\title{
Evolución y nuevas perspectivas del concepto de resiliencia: de lo individual a los contextos y a las relaciones socioeducativas
}

\section{Evolution and new perspectives of the concept of resilience: from an individual-based approach to socio-educational contexts and relationships}

\author{
Cristóbal Ruiz-Román ${ }^{1}$ \\ xtobal@uma.es \\ Jesús Juárez Pérez-Cea \\ juarez@uma.es \\ Lorena Molina Cuesta \\ lorenamolinacuesta@hotmail.com \\ Universidad de Málaga, España
}

\section{Resumen:}

La resiliencia es un concepto que en los últimos años está teniendo cada vez mayor presencia en los ámbitos sociales y educativos de nuestro país. Aunque su uso es relativamente reciente en el contexto español, sin embargo es un concepto que viene siendo utilizado desde el ámbito de la práctica profesional y avalado por numerosas investigaciones en el contexto anglosajón. Este trabajo tiene como objetivo estudiar la evolución que este concepto ha tenido durante las últimas décadas. Este análisis pone de manifiesto que la resiliencia es un tópico controvertido y en plena construcción. En efecto, los resultados del estudio bibliográfico y conceptual sobre la resiliencia muestran la evolución que ha tenido el tema en las

\begin{abstract}
:
Resilience is a concept that in recent years has had an ever-increasing presence in social and educational settings in Spain. Although its use is relatively recent in the Spanish context, it has been used in professional practice and supported by extensive research in the English-speaking context. This paper aims to study the evolution of this concept over the last decades. Our analysis found that resilience is a controversial topic and is still being constructed. The outcomes of the literature review and the conceptual study on resilience showed that this issue has been evolving over the last three decades, moving from an individual-based approach to resilience, focused on the qualities of a resilient person, towards a more socio-educational focus,
\end{abstract}

1 Dirección para correspondencia (correspondence address):

Cristóbal Ruiz-Román. Dpto. de Teoría e Historia de la Educación y Métodos de Investigación y Diagnóstico en Educación. Universidad de Málaga. Facultad de Ciencias de la Educación. Campus de Teatinos s/n. 29071. Málaga (España) 
Evolución y nuevas perspectivas del concepto de resiliencia: de lo individual a los contextos y a las relaciones socioeducativas

Cristóbal Ruiz-Román, Jesús Juárez Pérez-Cea y Lorena Molina Cuesta

tres últimas décadas desde un enfoque de la resiliencia centrado en el individuo y las cualidades de la persona resiliente, hacia un enfoque más socioeducativo, que se preocupa por comprender todos los elementos culturales y comunitarios que emergen e interactúan en los procesos resilientes. A partir de todo este análisis, el artículo arroja algunas conclusiones para repensar y actualizar el modo de abordar las prácticas e investigaciones sobre resiliencia.

\section{Palabras claves:}

Resiliencia; adversidad; relaciones educativas; comunidad; empoderamiento. concerned with understanding all the cultural and community-based elements that emerge and interact in resilience processes. From this analysis, the paper delimits some conclusions aimed at rethinking and updating how resilience practices and research are addressed.

\section{Keywords:}

Resilience; adversity; educational relationships; community; empowerment.

\section{Résumé:}

La résilience est un concept qui a une présence de plus en plus importante dans les domaines sociaux et éducatifs de notre pays. Même si son emploi est assez récent en Espagne, il s'agit d'un concept très fréquemment utilisé dans la pratique professionnelle et avalisé/approuvé par de nombreuses recherches en contexte anglo-saxon. La présente étude vise l'analyse de l'évolution que ledit concept a eue au cours des dernières décennies. Cette analyse met en évidence que la résilience est un cliché controversé et en pleine construction. En effet, les résultats de l'étude bibliographique et conceptuelle sur la résilience montrent l'évolution que ce sujet a eu pendant les trente dernières années à partir d'une approche basée sur l'individu et les qualités de la personne résiliente vers une approche plus socio-éducative, préoccupée par la compréhension de tous les éléments culturels et communautaires qui émergent et qui agissent dans les processus résilients. À partir de cette analyse, notre travail tire des conclusions pour repenser et actualiser la façon d'aborder les pratiques et les recherches sur la résilience.

\section{Mots-clés:}

Résilience; adversité; relations éducatives; communauté; autonomisation.

Fecha de recepción: 22-05-2016

Fecha de aceptación: 07-02-2017 


\section{Introducción: Un primer acercamiento a la idea de resiliencia}

El concepto de resiliencia deriva del latín "resiliere". Este concepto procede del ámbito de la física para designar la cualidad de los materiales con un alto grado de aguante y reposición ante un fuerte impacto. Así mismo este término también es utilizado en otras ramas como la medicina o la ecología. En medicina hace referencia a la habilidad que muestran ciertos huesos en los seres vivos para crecer en el sentido adecuado tras una fractura, en lugar de crecer de forma anómala dejando secuelas permanentes. Por su parte, en ecología, el concepto de resiliencia es utilizado para hacer referencia a la capacidad de las comunidades para resistir cambios propios de la naturaleza y evolucionar hacia un nuevo estado.

Este término empezó a utilizarse desde el ámbito de la psiquiatría en el mundo anglosajón - resilience - por científicos estadounidenses. Dichos investigadores comenzaron a estudiar diversos casos de niños en riesgo social que conseguían resistir, adaptarse y crecer con normalidad, a pesar de vivir en condiciones de pobreza, abandono y violencia. Estudios como los de Garmezy y Rutter, 1983; Anthony, 1974; Dugan y Coles, 1989; Gore y Eckenrode, 1994, observaron que hay algunos niños que han tenido trastornos psiquiátricos tras vivir eventos adversos o traumáticos, mientras que hay otros niños que tienen las mismas adversidades pero sin embargo las han superado.

Así pues, mientras que en el mundo anglosajón el concepto "resilien$\mathrm{ce}^{\prime \prime}$ viene siendo estudiado desde hace más de medio siglo y utilizado en las últimas tres décadas por los profesionales que trabajan con población en dificultad social (educadores, psicólogos, trabajadores sociales,...), en España, el concepto de resiliencia, es recientemente cuando se está empezando a introducir en los ámbitos de la investigación y la intervención socioeducativa.

En efecto, en el mundo anglosajón, la resiliencia se ha convertido en un concepto muy influyente en la intervención con las personas en dificultad social, hasta el punto de conformar un marco o paradigma de intervención para la promoción con diferentes estrategias y niveles de intervención (Jollien, 2000; Daniel, 2006 y 2010; Ungar, 2004; Hart y Heaver, 2013). En este sentido, la mayor parte de la literatura científica sobre resiliencia se encuentra principalmente en América del Norte debido, posiblemente, a que el concepto de resiliencia tiene una resonan- 
cia particularmente fuerte con la cultura norteamericana (Rigsby, 1994). Sin embargo, existe también una cantidad considerable de literatura en el Reino Unido, en los países nórdicos y en Australia (Rayner y Montague, 2000).

Si bien en estos países el estudio de la resiliencia empezó a desarroIlarse desde el ámbito de la psicología (Smith y Carlson, 1997), en las últimas décadas los trabajos sobre resiliencia se han extendido a los ámbitos social y educativo. En este sentido podemos hablar de publicaciones en relación al concepto de resiliencia desde problemáticas socioeducativas relacionadas con el abuso sexual (Spaccarelli y Kim, 1995); el maltrato infantil (Heller et al, 1999); los hijos de alcohólicos (Palmer, 1997); la crianza y cuidado de los niños (Gilligan, 1997); los niños con problemas emocionales y problemas de conducta (Lewis ,1999); la terapia familiar (Rutter, 1999); la promoción de niños vulnerables desde las escuelas (Raphael, 1993); la adopción (Clarke y Clarke, 2000), y en general, como un marco conceptual para la intervención socioeducativa (Newman y Blackburn, 2002).

En paralelo a todo este fluir, en los estudios sobre resiliencia han ido elaborándose algunos estudios longitudinales que han analizado la experiencia de personas durante un período de tiempo de su infancia hasta Ilegar a la edad adulta (Khan, 1964; Sobel, 1973, Werner y Smith, 1982). Dichos estudios constituyen una interesante fuente de información para analizar las variables personales, familiares y contextuales que se entretejen en la vida de las personas para que se produzcan procesos resilientes.

$\mathrm{Al}$ ir analizando las investigaciones sobre resiliencia se ponen de relieve dos grandes cuestiones sobre este concepto. Por un lado los estudios sobre resiliencia ponen de manifiesto que es un concepto complejo $y$, aunque en la literatura anglosajona lleva décadas manejándose dicho concepto, aún no hay un consenso y una definición cerrada sobre éste. Por otro lado, y a consecuencia del dinamismo del propio concepto, las investigaciones sobre resiliencia muestran una evolución del mismo. Dicha evolución muestra que el concepto en las tres últimas décadas ha ido desde un enfoque más psicológico de la resiliencia centrado en el individuo y las cualidades de la persona resiliente, hacia un enfoque más socioeducativo que trata de comprender todos los elementos culturales y comunitarios que emergen e interactúan en los procesos educativos resilientes. Parece pues oportuno realizar un análisis de la evolución en 
los estudios sobre resiliencia para explicitar de qué modo este concepto se ha ido construyendo hasta el momento.

\section{La evolución sobre los estudios de resiliencia: del individuo a la comunidad}

\subsection{Los primeros estudios sobre resiliencia: la resiliencia como característica individual}

En los primeros estudios sobre resiliencia, autores como Werner y Smith (1982) subrayan la importancia del carácter de ciertas personas capaces de mantener una actitud de supervivencia frente a la adversidad. Estos investigadores estudiaron los niños de una comunidad durante 30 años mediante un estudio longitudinal y prospectivo. Este estudio comenzó en 1955 en la ciudad hawaiana de Kauai, con niños que vivían diversas situaciones de vulnerabilidad social. Fue un trabajo basado en la observación de las cualidades que tenían estos niños y niñas para afrontar los hándicaps de vivir en una situación de exclusión social donde cualidades como la tolerancia, la capacidad de relacionarse, una buena autoestima, ilusión o sentido de la responsabilidad social fueron algunas de las características concluidas de esta investigación. Así mismo, en estos primeros compases del estudio de la resiliencia, tuvieron gran importancia las investigaciones de Rutter $(1979,1985)$, quien analizó características como el autocontrol, la autoeficacia, la capacidad de relación en las personas resilientes. Garmezy, Masten y Tellegen (1984), destacaron por ir definiendo las cualidades de las personas resilientes, entre las que destacaron el autocontrol, el sentido del humor, la capacidad crítica, la introspección y el autoconocimiento, la capacidad de resolución de conflictos, la autoestima y la efectividad. Por otra parte destacamos la línea de investigación ofrecida por Wolin y Wolin (1993), que describe cuales son los primeros pilares de la resiliencia. Así, partiendo del "Yo", definen una serie de características relevantes de las personas resilientes: la introspección, la independencia, la moralidad, la capacidad de relacionarse, el humor, la creatividad o iniciativa. 
Evolución y nuevas perspectivas del concepto de resiliencia: de lo individual a los contextos y a las relaciones socioeducativas

Cristóbal Ruiz-Román, Jesús Juárez Pérez-Cea y Lorena Molina Cuesta

Tabla 1

Elementos que emergen en la resiliencia según las primeras investigaciones sobre el tema

\begin{tabular}{|c|c|}
\hline AUTORES & CUALIDADES RESILIENTES DE SUS INVESTIGACIONES \\
\hline $\begin{array}{l}\text { Werner y Smith } \\
(1982)\end{array}$ & $\begin{array}{l}\text { - Responsabilidad social } \\
\text { - Tolerancia } \\
\text { - } \text { Capacidad de comunicación } \\
\text { - Autoestima } \\
\text { - Ilusión en nuevas metas y objetivos }\end{array}$ \\
\hline $\begin{array}{l}\text { Rutter } \\
(1979 \text { y } 1985)\end{array}$ & $\begin{array}{l}\text { - Autocontrol } \\
\text { - } \text { Autoeficacia } \\
\text { - Capacidad de relación } \\
\text { - Búsqueda de tutores resilientes }\end{array}$ \\
\hline $\begin{array}{l}\text { Garmezy, Masten y Tellegen } \\
\text { (1984) }\end{array}$ & $\begin{array}{l}\text { - Personas efectivas } \\
\text { - } \text { Buenas perspectivas } \\
\text { - Autoestima } \\
\text { - } \text { Capacidad de resolución de conflictos } \\
\text { - Introspección y autoconocimiento } \\
\text { - Autocontrol } \\
\text { - Sentido del humor } \\
\text { - Capacidad crítica }\end{array}$ \\
\hline $\begin{array}{l}\text { Wolin y Wolin } \\
(1993)\end{array}$ & $\begin{array}{ll}\text { - } & \text { Introspección } \\
\text { - Independencia } \\
\text { - } \text { Capacidad de relacionarse } \\
\text { - } \text { Moralidad } \\
\text { - Humor } \\
\text { - } \text { Creatividad } \\
\text { - Iniciativa } \\
\end{array}$ \\
\hline
\end{tabular}

En definitiva, como se puede observar en la Tabla 1, en los primeros estudios sobre el concepto de resiliencia, estos estaban ligados a cualidades del sujeto resiliente y todas las investigaciones tienen un denominador común: identificar o definir cuáles son esas características personales. Podemos observar que existen interesantes coincidencias entre estas primeras investigaciones. Así es interesante las similitudes entre el estudio de Werner y Smith (1982) y el de Garmezy, Masten y Tellegen (1984), donde la autoestima o la capacidad para relacionarse, ya sea como simple comunicación o términos de resolución de conflictos, tienen cierta relevancia. A esta última característica también dan importancia Wolin y Wolin (1993) en su estudio. Por otra parte, tanto para Rutter (1979, 1985) como para Garmezy, Masten y Tellegen (1984), la capacidad de con- 
trolarse uno mismo en momentos de tensión es otra característica compartida en sus estudios para definir al sujeto resiliente. Al tiempo, estos autores coinciden con Wolin y Wolin (1993) al hablar de la capacidad de conocerse y hacer un análisis interno de uno mismo cuando hablan de aspectos como autoconocimiento e introspección.

Como se puede apreciar, en esta primera etapa el concepto resiliencia está muy arraigado a aquellas características personales del individuo. Sin embargo, Rutter $(1979,1985)$ aporta una clave que luego será útil para abrir la puerta a una segunda etapa en los estudios sobre resiliencia. En efecto, en los trabajos de Rutter se comienza a hablar de la búsqueda de tutores resilientes y con ello se abre una nueva vía para explorar elementos ajenos al individuo que generan resiliencia.

\subsection{Una nueva fase en los estudios de resiliencia: el paso de identificar cualidades personales a explorar la influencia del contexto}

Los estudios sobre la resiliencia entran en una nueva fase cuando dan tanta importancia a la identificación de cualidades y características personales como a la comprensión de cómo y dónde se obtienen estas características resilientes. En esta época de la década de los 90 siguen siendo de interés los aportes de Rutter (1999) y Masten (2001) que paulatinamente van mostrando dicha evolución en sus estudios. A estos estudiosos que ya venían realizando investigaciones sobre resiliencia, se añaden otros que amplían las perspectivas del abordaje de la resiliencia desde la perspectiva que se ha señalado. Entre ellos podemos citar a Benson (1997) y Luthar (2003).

Estos vienen a incidir en su preocupación por una idea: conocer la influencia que tiene el entorno en la posibilidad de que las personas sean resilientes. En líneas generales, esta forma de abordar la resiliencia se encuentra estrechamente vinculada al modelo ecológico propuesto por Bronfenbrenner (1987), quien propugna la influencia que tienen los entornos y ambientes en el desarrollo del sujeto.

En este sentido, Masten y Obradovic (2006), elaboran un modelo eco-sistémico de resiliencia humana donde en primer lugar sitúan el cerebro humano, que es el que procesa la información, fomenta diferentes habilidades en la resolución de los problemas y tiene la flexibilidad o plasticidad de nuestro raciocinio. Siguiendo el esquema que se ofrece en la Figura 1 aportan una serie de elementos que consideran importantes 
en la construcción de las capacidades resilientes. Así, elementos como el apego seguro, el sistema familiar, los grupos de pares o iguales o la propia cultura y la sociedad, se van propugnando como elementos vertebradores de las posibilidades de ser resiliente de una persona.

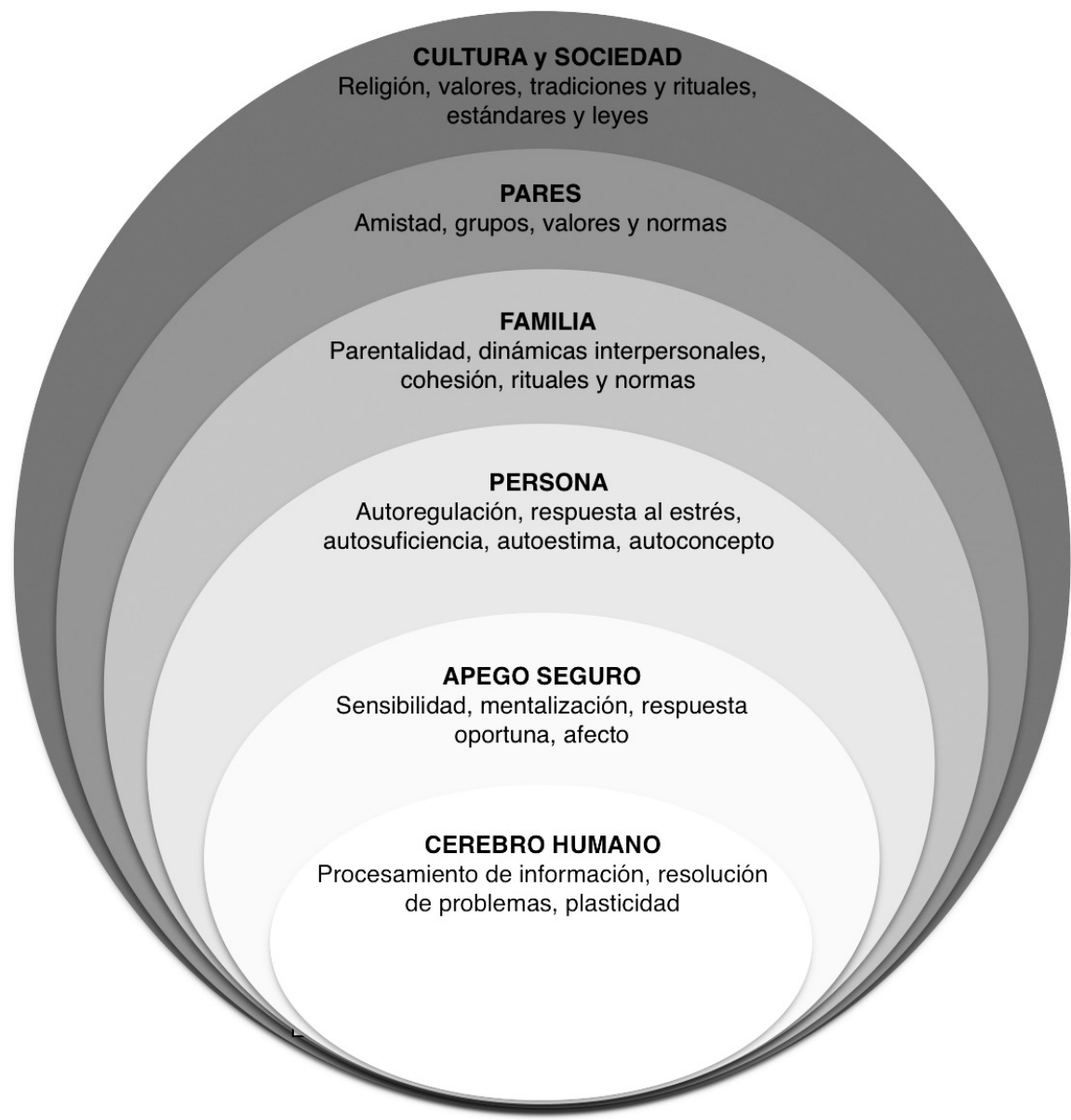

Figura 1: Modelo eco-sistémico de resiliencia humana desarrollado por Masten y Obradovic (2006)

\subsection{El cambio de paradigma: De la persona resiliente al proceso resiliente}

Las investigaciones agrupadas en la etapa anterior inciden en su preocupación por comprender los elementos del entorno que ayudan a cons- 
truir a la persona resiliente. Sin embargo, dichas investigaciones siguen manteniendo el concepto de resiliencia ligado a las capacidades de la persona. La resiliencia, aún condicionada por el contexto, se sigue ubicando en la persona. No obstante los avances de estos investigadores, que siguen los postulados de la Teoría de Bronfenbrenner, son la antesala de un nuevo modo de explorar la resiliencia. Una forma de abordar la resiliencia que va más allá de reducirla a una cualidad del individuo condicionada por el contexto. Si la resiliencia se construye entre el individuo y el entorno, la resiliencia no solo se ubica en la persona, sino también entre las personas y los contextos que habitan (Masten y Obradovic, 2006; Ungar, Ghazinour y Richter, 2013; Ungar, 2015). De ese modo la resiliencia transita de una cualidad a un proceso, de un calificativo a una acción. Un proceso del cual es agente el individuo pero también otros elementos a los que se empieza a prestar cada vez mayor atención. Efectivamente autores como Madariaga (2014) empiezan a entender que la resiliencia es un proceso en el que intervienen múltiples agentes, entre ellos claro está el individuo, pero un proceso que desborda al individuo al accionarse también desde otros agentes y contextos. La resiliencia es un proceso que no está solo en el sujeto sino que se va conformando entre el sujeto, las posibilidades que ofrece el entorno y los contextos, así como en las relaciones educativas que se generan entre éstos (Ungar, 2015). Así, la resiliencia desde una concepción sistémica o procesual, transciende los límites de una concepción individualista y abre un nuevo foco de atención hacia la cultura, la comunidad y las relaciones socioeducativas (Costa, Forés y Burguet, 2014), como elementos a tener en cuenta en los procesos resilientes.

La tesis principal que defienden las investigaciones de este tercer enfoque se centran entonces en pasar de entender la resiliencia como una cualidad o un adjetivo, a entenderla como un proceso o una acción. Sería algo así como lo que ocurre con el concepto de educación. Se puede decir que una persona tiene educación. Educación se convierte entonces en una cualidad. Pero la cuestión es que esta idea de educación como resultado es fruto de una acción previa, la educación como acción educativa (Esteve, 1983; Castillejo, 1994). En términos de resiliencia ocurriría algo similar. Cuando se habla de una persona resiliente, la resiliencia es entendida como una cualidad que tiene la persona, sin embargo, para que la resiliencia llegue a ser entendida como un resultado o adjetivo -persona resiliente- antes ha debido producirse todo una 
serie de acciones o procesos (dentro y fuera de la persona) que al final den como resultado una persona resiliente. Pero la resiliencia como proceso es más que un resultado concretizado en una persona resiliente, al igual que la educación como proceso es mucho más que una persona educada. Así pues, aunque la resiliencia pueda ser entendida como un resultado y se hable de persona resilientes, como se habla de personas educadas, tiene un interés extra, trascender esta concepción de la resiliencia como resultado en una persona y entender la resiliencia como una acción.

En esta nueva teoría se comienza a entender que "la resiliencia es un proceso en constante desarrollo (...) la resiliencia es un capítulo de teorías del vínculo" (Madariaga, 2014, 33). Así pues, en este nuevo enfoque se resaltan dos grandes ideas que suponen una evolución con respecto a enfoques anteriores y otorga nuevas miras al término resiliencia. Por un lado el concepto proceso, entendiendo que la resiliencia no es una capacidad o conjunto de capacidades, sino que es un proceso o conjunto de acciones en las que las personas van interactuando con otros agentes de resiliencia. Y por otro lado la idea de vínculos y de relaciones basadas en la afectividad. Y es que "educadores, miembros de la familia, maestros, amigos podían ser seleccionados como tutores de resiliencia, puntos de apoyo a quien aferrarse para iniciar la reconstrucción en caso de encontrarse una situación difícil" (Rubio y Puig, 2015, 81). Así desde este nuevo enfoque se abre una perspectiva hacia la multidimensionalidad de la resiliencia entendiendo como agentes activos de este proceso al entorno, sus condiciones materiales y culturales, las instituciones y los propios vínculos como espacios de interrelación donde germina la resiliencia.

\section{Elementos constituyentes de los procesos resilientes}

Una vez abierta esta concepción de la resiliencia como proceso en lugar de como cualidad, parece oportuno abordar los elementos que los estudios más recientes señalan que están presentes en los procesos resilientes. Con ello, se observará que las investigaciones vienen a reubicar la resiliencia más allá del individuo y de sus capacidades, en otros elementos o agentes externos a la persona que ayudan a florecer los procesos resilientes. 


\subsection{Las cualidades personales como agentes de resiliencia}

Abordar la resiliencia desde un punto de vista más amplio, analizándola como un proceso y no como una cualidad individual de la persona, no impide confirmar que hay características individuales de la persona que influyen positivamente en los procesos resilientes. Tales características, que ya fueron identificadas por las primeras investigaciones sobre resiliencia -y descritas en los apartados anteriores de este trabajo-, no son innatas, sino que se pueden adquirir a lo largo de la vida, se pueden aprender y enseñar al igual que la resiliencia. Características como la introspección o el autoconocimiento, la aceptación de uno/a mismo/a, la autoestima, la autonomía, las habilidades sociales, la empatía, la capacidad de iniciativa, la motivación, el humor, la creatividad, la capacidad de pensamiento crítico, la capacidad para descubrir un sentido, la inteligencia emocional (Wolin y Wolin, 1993; Vanistendael y Lecomte, 2002; Sanz, 2002; Cyrulnik, 2002; Forés y Grane, 2008 y Manciaux, 2010) todas ellas son características individuales, que como se ha venido estudiando desde los orígenes de las investigaciones sobre resiliencia, pueden estar presentes en los procesos resilientes. $Y$ es conveniente reiterar que pueden estar presentes, puesto que no son necesarias todas ellas para que surjan los procesos resilientes, al tiempo que sería difícil encontrar a la persona-tipo que reuniera todas estas cualidades.

\subsection{Las condiciones materiales: la base de los procesos resilientes}

Las necesidades físicas básicas constituyen la base sobre la que está construida la resiliencia. Para Vanistendael y Lecomte (2002), esas necesidades básicas pueden ser aquéllas como la alimentación, el sueño, el descanso y los cuidados básicos para la salud, etc. En este sentido, y por ilustrar estas condiciones materiales y básicas, se puede citar el estudio de Fiorentino, que hace referencia a un programa dirigido a niños y niñas de la calle, donde se encontró "que la necesidad de dormir debía ser satisfecha antes que ninguna otra, debido a la explotación que se hacía de los mismos a nivel laboral." (2008, 104). De modo que no se podía trabajar la recuperación psicológica de estos niños y niñas hasta que la recuperación física (sueño y alimentación) no se diera por finalizada. En efecto, como señalan Hart y Heaver (2013), este hecho refleja que si las necesidades básicas no están cubiertas es muy complicado incidir 
en los demás aspectos. En esta línea, para que se desarrollen procesos resilientes, las actuales investigaciones inciden en la necesidad de que estos han de constituirse sobre condiciones básicas y materiales, como una vivienda digna o la alimentación (Vanistendael y Lecomte, 2002) que hagan factibles dichos procesos resilientes.

\subsection{El factor tiempo en los procesos resilientes}

A la hora de analizar los procesos resilientes, la dimensión temporal es un elemento al que en los últimos años se le está presentando un interés extraordinario, por cuanto los estudios están poniendo de relieve que el tiempo tiene importantes implicaciones sobre los procesos resilientes a distintos niveles.

En primer lugar, y a efectos de etapas vitales, Manciaux (2010) expone que la mayoría de las investigaciones sobre resiliencia solían tomar como referencia las experiencias vividas de niños/as y adolescentes, y rara vez sobre adultos/as o personas mayores. Sin embargo, esta escasez de estudios sobre los procesos resilientes en estas edades no quiere decir que la resiliencia no pueda desarrollarse a lo largo de toda la vida y que en la edad adulta o en la vejez no puedan producirse tales procesos. "Los traumas graves no perdonan a la tercera edad. Sea una enfermedad, deficiencias ligadas al envejecimiento o bien por problemas sociales [...], pueden perturbar gravemente a muchas personas que se hacen mayores." (Manciaux, 2010, 106). Así pues, la resiliencia no aparece solo en las primeras etapas de la vida, sino que es un proceso vital que puede aparecer en cualquier momento (Padilla et al, 2019).

En segundo lugar, el tiempo juega un importante papel en la duración de la adversidad. No tiene la misma duración un terremoto, que es un acontecimiento que sucede en un instante puntual a pesar de sus consecuencias, que el nacer y vivir en un barrio marginal durante toda una vida. Es decir, dependiendo del tiempo que dure la adversidad, los procesos resilientes también se irán fraguando de una u otra manera (Madariaga, 2014).

Y por último, y en relación a lo anterior, los elementos o agentes que intervienen en los procesos resilientes son aspectos que necesitan tiempo para que se consoliden. En efecto, tanto el aprendizaje de ciertas actitudes individuales resilientes, como la construcción de redes sociales en torno al individuo que promuevan procesos resilientes necesitan 
del tiempo para forjarse. Así es, los elementos claves en los procesos de resiliencia necesitan de tiempo para tomar forma y afianzarse como agentes generadores de resiliencia ante posibles adversidades (Hart y Heaver, 2013).

\subsection{La construcción de procesos resilientes en las comunidades y entre las comunidades}

Como se ha venido exponiendo a lo largo de estas páginas, tras varias décadas de estudios sobre resiliencia, las investigaciones actuales apuestan por una mirada más amplia y renovada, al analizar la resiliencia "no como una coraza personal de protección, sino como un engranaje relacional y eco-sistémico que permite encontrar oportunidades donde podría darse el estancamiento o deterioro" (Gómez y Kotliarenco, 2010, 105). De esta manera surgen los estudios que ponen el foco de atención en la(s) comunidad(es) como generadora(s) de resiliencia (Hart y Heaver, 2013; Ungar, Ghazinour y Richter, 2013; Madariaga, 2014).

Es dentro de la comunidad donde las personas afrontan las adversidades y por tanto es el espacio donde se crean las bases de los procesos resilientes (Soler, Trilla, Jiménez y Úcar, 2017). La comunidad "es capaz de utilizar los recursos y las capacidades necesarias para afrontar las adversidades" (Forés y Grané, 2008, 105).

La comunidad posee algunos elementos sin los cuales serían inviables los procesos resilientes. Pero sin lugar a dudas el principal de estos elementos de las comunidades es la propia cultura de los grupos humanos. En la cultura es donde emerge la posibilidad para dar significación a las adversidades, trascenderlas y elaborar estrategias y vías para superarlas (Geertz, 1990; Bruner, 1997). En este sentido, de la propia cultura de las comunidades es de donde surge la resistencia grupal ante la adversidad y la solidaridad ante esta, que prestan apoyo y protección a los propios miembros de la comunidad (Ruiz-Román, Calderón y Torres, 2014).

En este sentido, el estudio de Ruiz-Román, Calderón y Juárez (2017) señala que cuando las personas están expuestas a situaciones de riesgo o ante determinadas adversidades, la comunidad puede llegar a promover la solidaridad para que la situación adversa desaparezca, para que pueda generarse una cultura de lucha y cooperación ante la adversidad. En dicho proceso en el que la comunidad da significado a una adversidad, 
la propia comunidad sufre una transformación grupal ante esas adversidades. Como señalan Suárez-Ojeda y Autler, "las desgracias pueden convertirse en un desafío que moviliza las capacidades de la población para solidarizarse y renovarse, no solo a nivel de infraestructura sino también del tejido social" $(2003,273)$.

Una de las principales formas en la que se concretan las comunidades es la familia. La familia construye una red de significados colectivos que ayudan a dotar de sentido no solo las experiencias colectivas, sino también las experiencias personales (Esteve, Ruiz-Román y Rascón, 2008). Dicha red de significados al mismo tiempo ayudan a mantener su integridad como unidad ante las adversidades, desarrollando competencias positivas para que esas situaciones difíciles, más allá de romper la unión familiar, sean interpretadas como situaciones que han de afrontarse agrupadamente. De este modo, y como señalan los recientes trabajos de Becvar (2012) y Walsh (2015), los procesos resilientes pueden aparecer con mayor facilidad si la familia actúa como un agente facilitador. Una familia que construye resiliencia hace que ya no solo sea el individuo, sino toda la composición familiar, la que crea una serie de valores y significados sobre los que se asienta la resiliencia: responsabilidad, resistencia ante la adversidad, compasión, apoyo mutuo, posibilidad de comunicación, amor, respeto y un ambiente de afecto y equilibrio emocional tal y como indican en su trabajo Allan y Ungar (2014). Así pues se puede hablar de la familia que promueve la resiliencia como aquélla "que crea formas activas, saludables y sensibles de satisfacer las necesidades y el desarrollo de cada uno de los componentes" (Forés y Grané, 2008, 100).

Al igual que la familia es importante para promover procesos resilientes, recientes investigaciones también reivindican el papel fundamental que juegan en dichos procesos resilientes otras redes de apoyo socioeducativo más allá de la familia (Civís y Longás, 2015, Azorín, 2017; Ruiz-Román, Molina y Alcaide, 2019). Con estas redes de apoyo se hace referencia a las diversas comunidades socioeducativas, grupos, agentes o instituciones en los que se hacen presentes procesos educativos de una manera formal o no formal (Arnaiz, 2012, Herrera, Juárez y Ruiz-Román, 2019). Efectivamente, en estos ambientes se pueden generar procesos resilientes, dado que pueden constituirse en estructuras de apoyo y contextos privilegiados para la construcción de la resiliencia (Uriarte, 2006). Estas comunidades socioeducativas son necesarias en todas las etapas 
de la vida, y si se establece un clima de confianza y afecto, se convierten en un espacio donde las personas se sienten reconocidas, cómodos/as, respetados/as y queridos/as (Torres, 2012; Hart y Heaver, 2013).

\subsection{Las relaciones educativas: el enlace en el que se concibe la resiliencia}

Con el análisis realizado en este trabajo acerca de la evolución del concepto de resiliencia, se han ido analizando los elementos que contribuyen a que una persona salga recuperada de una situación adversa en un momento determinado de su vida. Más arriba se mencionaban las características individuales de la persona que afronta la adversidad, las necesidades básicas, el tiempo y las comunidades y redes de apoyo como los elementos que van constituyendo los procesos resilientes. En este último apartado, se abordará un aspecto más que, según los estudiosos contemporáneos de la resiliencia, forma parte de la sinergia resiliente que crece alrededor de la persona herida. Este último elemento no es otro que la relación, como lugar en el que se van fraguando los procesos resilientes (Cyrulnik, 2004).

Cuando una persona experimenta una situación adversa y encuentra el acompañamiento de otra en esos momentos de dificultad, se generan relaciones. Relaciones que Vanistendael (2005) en sus trabajos los denomina como encuentros, donde la persona humana es aceptada por otra persona. En estos encuentros resilientes según (Bermejo, 2007) ha de brindarse un amor incondicional y se crea un clima de confianza donde las personas se sienten seguros/as y respetados/as. Ser reconocido/a por otra persona hace que la autoestima mutua y la confianza aumente (Cisternas y Quintana, 2018). De este modo, la persona herida se sentirá querida y valorada cuando más lo necesita, sin temor a avergonzarse por el sufrimiento que ha padecido, y poder convertir la vergüenza en orgullo (Cyrulnik, 2004).

Este proceso de acompañamiento se convierte en un proceso educativo que rompe moldes, desafía las interpretaciones hegemónicas sobre la realidad y genera procesos resilientes de empoderamiento y libertad. En estos casos, no solo es un acompañamiento incondicional con la persona, sino que al tiempo el acompañamiento constituye una subversión contra la adversidad (Ruiz-Román, Calderón y Juárez, 2017). Como Cyrulnik $(2002,2004)$ argumenta persistentemente en sus trabajos, el encuentro engendra un proceso resiliente, que traerá consigo un 
subversivo renacer tras el trauma. En efecto, en este encuentro se construye otro discurso social: "Consigo que me quieran por la totalidad de lo que soy" (Cyrulnik, 2004, 27). Esta transformación viene provocada por el discurso íntimo de la persona como consecuencia del efecto del discurso social y viceversa. Un discurso social que brota de este encuentro resiliente y que va dando significado y tomando postura ante la adversidad. En efecto, el encuentro resiliente crea un relato común, un frente común en la tarea de transformar la adversidad. Así, en los encuentros resilientes, en lo comunitario, se van creando contra-relatos. Contrarrelatos resilientes que más allá de una estrategia socioeducativa de acompañamiento y empoderamiento del individuo, terminan por convertirse en una forma de reinterpretar y afrontar colectivamente las adversidades.

\section{Conclusiones}

A lo largo de este artículo se ha realizado un análisis de la evolución del concepto resiliencia. Dicha evolución pone de relieve que los primeros estudios sobre resiliencia se centraron fundamentalmente en conocer las capacidades de la persona resiliente. Posteriormente, las investigaciones sobre este controvertido concepto ya no solo se ocuparon de estudiar a la persona resiliente, sino de comprender los procesos resilientes, poniendo de ese modo el foco de atención en qué aspectos y contextos influyen para la aparición de dichos procesos. En este sentido, las últimas investigaciones sobre resiliencia ponen en evidencia que este concepto está en plena construcción. En efecto, todo el entramado que conlleva el concepto de resiliencia nos habla de un concepto que sigue radicalmente abierto a nuevas vías de investigación.

Metafóricamente podríamos describir dicha complejidad del concepto de resiliencia con el concepto de red. Los procesos resilientes son complejos y las personas que vivencian una situación adversa se encuentran en medio de esa red. Esa red está compuesta por una gran cantidad de elementos que sostienen a la persona: la familia, la comunidad, una relación educativa, el grupo de iguales, agentes educativos formales o no formales, la cultura, los relatos personales o comunitarios. Todos ellos son elementos que de una u otra manera van generando sinergias. Sinergias que ponen en marcha procesos resilientes. Esas sinergias, 
actúan a modo de nodos dentro de la red, y con ello se convierten en verdaderos agentes de resiliencia.

Con todo ello, podemos concluir que los estudios sobre resiliencia están ante un nuevo reto: el analizar y comprender cómo se generan los procesos resilientes, cómo se fragua la compleja red de la resiliencia y cuáles son sus nodos y sinergias. Analizar el funcionamiento de todo este complejo entramado que se construye alrededor de un proceso resiliente es a todas luces imprescindible para tener ciertas y mejores claves para el trabajo socioeducativo. Un trabajo socioeducativo que vaya más allá de lo individual y recoja las sinergias que hay en lo común, para de ese modo fortalecer los procesos resilientes.

\section{Referencias}

Allan, R. y Ungar, M. (2014). Developing a measure of fidelity for an ecological approach to family therapy. Journal of Family Psychotherapy, 25(1), 26-41.

Anthony, E.J. (1974). Introduction: The syndrome of the psychologically vulnerable child. In Anthony, E.J. y Koupernik, C. (Ed.), The child in his family: Children at Psychiatric Risk. Vol. 3. New York: Wiley, 3-10.

Arnaiz, P. (2012). Escuelas eficaces e inclusivas cómo favorecer su desarrollo. Educatio Siglo XXI, 30 (1), 25-44.

Azorín, C. (2017). Redes de colaboración entre escuelas inglesas para la mejora de la inclusión socioeducativa. Profesorado. Revista de Currículum y Formación del Profesorado, Número Extraordinario, 29-48.

Becvar, D. S. (Ed.). (2012). Handbook of family resilience. New York: Springer Science y Business Media.

Benson, P. L. (1997). All kids are our kids: What communities must do to raise caring and responsible children and adolescents. San Francisco: Jossey-Bass.

Bermejo, J.C. (2007). La relación de ayuda en el ámbito educativo. Santander: Sal Terrae.

Bronfenbrenner, U. (1987). La ecología del desarrollo humano: experimentos en entornos naturales y diseñados. Barcelona: Paidós.

Bruner, J. (1997). La educación, puerta de la cultura. Madrid: Visor.

Castillejo, J.L. (1994). La educación como fenómeno, proceso y resultado, en AAVV, Teoría de la Educación. Madrid: Taurus. 15-28.

Cisternas, A. y Quintana, J. (2018). Educación relacional. Diez claves para una pedagogía del reconocimiento. Madrid. SM

Civís, M. y Longás, J. (2015). La colaboración interinstitucional como respuesta al desafío de la inclusión socioeducativa. Análisis de 4 experiencias de trabajo en red a nivel local en Cataluña. Educación XX1, 18(1), 213-236.

Clarke, A. y Clarke, A. (2000). Early Experience and the Life Path. London: Jessica Kingsley. 
Evolución y nuevas perspectivas del concepto de resiliencia: de lo individual a los contextos y a las relaciones socioeducativas

Cristóbal Ruiz-Román, Jesús Juárez Pérez-Cea y Lorena Molina Cuesta

Costa, S., Forés, A. y Burguet, M. (2014). Els tutors de resiliencia en l'educació social. Temps d'Educació, 46, 91-106.

Cyrulnik, B. (2002). Los patitos feos. La resiliencia: una infancia infeliz no determina la vida. Barcelona: Gedisa.

Cyrulnik, B. (Comp.) (2004). El realismo de la esperanza. Testimonios de experiencias profesionales en torno a la resiliencia. Barcelona: Gedisa.

Daniel, B. (2006). Operationalizing the concept of resilience in child neglect: case study research. Child: Care, Health and Development, 32(3), 303-309.

Daniel, B. (2010). Concepts of adversity, risk, vulnerability and Resilience: A discussion in the context of the 'Child Protection System'. Social Policy and Society, 9(2), 231241.

Dugan, T. y Coles, R. (1989). The child our times. New York: Brunner.

Esteve, J.M. (1983). El concepto de educación y su red nomológica, en AAVV, Teoría de la Educación. Murcia: Límites. 11-25.

Esteve, J. M., Ruiz, C. y Rascón, M. T. (2008). La construcción de identidad en los hijos de inmigrantes marroquíes. Revista Española de Pedagogía, 241, 489-508.

Fiorentino, M. T. (2008). Construcción de la resiliencia en el mejoramiento de la calidad de vida y la salud. Suma Psicológica, 15(1), 95-114.

Forés, A. y Grané, J. (2008). La resiliencia: Crecer desde la adversidad. Barcelona: Plataforma Actual.

Garmezy, N. y Rutter, M. (1983). Stress, coping, and development in children. New York: McGraw-Hill.

Garmezy, N., Masten, A. S. y Tellegen, A. (1984). The study of stress and competence in children: A building block for developmental psychopathology. Child Development, 55, 97-111.

Geertz, C. (1990). La interpretación de las culturas. Barcelona: Gedisa.

Gilligan, R. (1997). Beyond Permanence? The importance of resilience in child placement practice and planning. Adoption and Fostering, 21(1), 12-20

Gómez, E. y Kotliarenco, M. A. (2010). Resiliencia Familiar: Un enfoque de investigación e intervención con familias multiproblemáticas. Revista de Psicología, 19(2), 103-132.

Gore, S. y Eckenrode, J. (1994). Context and process in research on risk and resilience, in: R. Haggerty et al. (Eds) Stress, Risk and Resilience in Children and Adolescents: Processes, Mechanisms and Interventions. New York: Cambridge University Press.

Hart, A. y Heaver, B. (2013). Evaluating resilience-based programs for schools using a systematic consultative review. Journal of Child and Youth Development, 1(1), 27-53.

Heller, S.S., Larrieu, J.A., D'Imperio, R. y Boris, N.W. (1999). Research on resilience to child to child maltreatment. Abuse and Neglect, 23, 321-338.

Jollien, A. (2000). Eloge de la faiblesse. Paris: Le Cerf.

Herrera, D., Juárez, J. Y Ruiz-Román, C. (2019). Collaborative leadership to subvert marginalisation: the workings of a socio-educational network in Los Asperones, Spain. School Leadership \& Management, 40:2-3, 203-220.

Khan, M. (1964). The concept of cumulative trauma. Psychoanalytical Study of the Child. New York: International Universities Press. 
Evolución y nuevas perspectivas del concepto de resiliencia: de lo individual a los contextos y a las relaciones socioeducativas

Cristóbal Ruiz-Román, Jesús Juárez Pérez-Cea y lorena Molina Cuesta

Lewis, J. (1999). Research into the concept of resilience as a basis for the curriculum for children with EBD. Emotional and behavioural difficulties, 4(2), 11-22.

Luthar, S.S. (2003). Resilience and Vulnerability. New York: Cambridge University Press

Madariaga, J.M. (2014). Nuevas miradas sobre la resiliencia: ampliando ámbitos y prácticas. Barcelona: Gedisa.

Manciaux, M. (Comp.) (2010). La resiliencia: resistir y rehacerse. Barcelona: Gedisa.

Masten, A.S. (2001). Ordinary magic: resilience processes in development. American Psychologist, 56(3), 227-238.

Masten, A. y Obradovic, J. (2006). Competence and resilience in development. Annals of the New York Academy of Sciences, 1094(1), 13-27.

Newman, T. y Blackburn, S. (2002). Transitions in the Lives of Children and Young People: Resilience Factors. Edinburgh: Scottish Executive.

Padilla, M., Ruiz-Román, C., Pérez-Ruiz, E., Rueda, A., Redondo, M. \& Rivas-Ruiz, F. (2019). Clinical and sociodemographic factors that may influence the resilienceof women surviving breast cancer: cross-sectional study. Supportive Care in Cancer, 27 (4), 1279-1286.

Palmer, N. (1997). Resilience in adult children of alcoholics: A nonpathological approach to social work practice. Health y Social Work, 22(3), 201-209.

Raphael, B. (1993). Adolescent resilience: the potential impact of personal development in schools. Journal of paediatrics and child health, 29(s1), S31-S36.

Rayner, M., y Montague, M. (2000). Resilient children and young people. Melbourne, Vic.: Deakin University, Policy and Practice Unit.

Rigsby, L. C. (1994). The Americanization of resilience: deconstructing research practice, in M. C. Wangand E. W. Gordons (eds.) Educational Resilience in Inner-City America: Challenges and Prospects. NJ: Lawrence Erlbaum.

Rubio, J.L. y Puig, G. (2015). Tutores de Resiliencia. Barcelona: Gedisa.

Ruiz-Román, C., Calderón, I. y Torres, F. J. (2014). Construir la identidad en los márgenes de la globalización: educación, participación y aprendizaje. Cultura y Educación, 23(4), 589-599.

Ruiz-Román, C., Calderón, I. y Juárez, J. (2017). La resiliencia como forma de resistir a la exclusion social: un análisis comparativo de casos. Pedagogía Social. Revista Interuniversitaria, 29, 129-141.

Ruiz-Román, C., Molina, L. y Alcaide, R. (2018). Trabajo en red y acompañamiento socioeducativo con estudiantes de secundaria en desventaja social. Profesorado. Revista de Curriculum Formación del Profesorado, 22 (3), 453-474.

Ruiz-Román, C., Molina, L. \& Acaide, R. (2019). We have a common goal: Support Networks for the educational and social development of Children in Disadvantaged Areas. British Journal of Social Work, 49 (6), 1658-1676.

Rutter, M. (1979). Protective factors in children's responses to stress and disadvantage. In M.W. Kent y J.E. Rolf (Eds.), Primary prevention in psychopathology: Social competence in children. Hanover, NH: University Press of New England.

Rutter, M. (1985). Resilience in the face of adversity: protective factors and resistance to psychiatric disorders. British Journal of Psychiatry, 147, 589-611. 
Evolución y nuevas perspectivas del concepto de resiliencia: de lo individual a los contextos y a las relaciones socioeducativas

Cristóbal Ruiz-Román, Jesús Juárez Pérez-Cea y Lorena Molina Cuesta

Rutter, M. (1999). Resilience concepts and fi ndings: implications for family therapy. Journal of Family Therapy, 21, 119-160.

Sanz, J. (2002). El humor como valor terapéutico. Med Clin (Barc), 119(19), 734-737.

Smith, C., y Carlson, B. E. (1997). Stress, coping, and resilience in children and youth. The Social Service Review, 231-256.

Sobel, R. (1973). What went right? The natural history of the early traumatized, in E.G. Witenberg (ed.), Interpersonal explorations in psychoanalysis. New York: Basic.

Soler, P., Trilla, J., Jiménez, M, y Úcar, X. (2017). La construcción de un modelo pedagógico del empoderamiento juvenil: espacios, momentos y procesos. Pedagogía Social. Revista Interuniversitaria, 30, 19-34.

Spaccarelli, S. y Kim, S. (1995). Resilience criteria and factors associated with resilience in sexually abused girls. Child Abuse y Neglect, 19(9), 1171-1182.

Suárez-Ojeda, N. E. y Autler, L. (2003). La resiliencia en la comunidad: un enfoque social. En E. H. Grotberg (Ed.), La resiliencia en el mundo de hoy. Cómo superar las adversidades. Barcelona: Gedisa. 271-300.

Torres, J.A. (2012). Estructuras organizativas para una escuela inclusiva: promoviendo comunidades de aprendizaje. Educatio Siglo XXI, 30 (1), 45-70.

Ungar, M. (2004). The importance of parents and other caregivers to the resilience of high-risk adolescents. Family Process, 43(1), 23-41.

Ungar, M. (2015). Practitioner Review: Diagnosing childhood resilience: A systemic approach to the diagnosis of adaptation in adverse social ecologies. Journal of Child Psychology and Psychiatry, 56(1), 4-17.

Ungar, M., Ghazinour, M., y Richter, J. (2013). What is Resilience Within the Ecology of Human Development? Journal of Child Psychology and Psychiatry, 54 (4), 348-366.

Uriarte, J. (2006). Construir la resiliencia en la escuela. Revista de Psicodidáctica, 11(1), 7-24.

Vanistendael, S. (2005). La resiliencia: Desde una inspiración hacia cambios prácticos. II Congreso Internacional de los trastornos del comportamiento en niños y adolescentes. Recuperado el 1 de noviembre de 2015 de, https://www.obelen.es/upload/262D. pdf

Vanistendael, S. y Lecomte, J. (2002). La felicidad es posible. Despertar en niños maltratados la confianza en sí mismos: construir la resiliencia. Barcelona: Gedisa.

Walsh, F. (2015). Strengthening family resilience. New York: Guilford Publications.

Werner, E. E. y Smith, R. S. (1982). Vulnerable but invincible. A longitudinal study of resilient children and youth. New York: McGrawHill.

Wolin, S. J. y Wolin, S. (1993). The resilient self. New York: Willard. 